\title{
Educational Software Tool for Removing Items from a One-Dimensional Array
}

Adrian LUPASC ${ }^{\star}$

\begin{tabular}{l}
\hline \multicolumn{1}{c}{ A R T I C L E I N F O } \\
\hline Article history: \\
Accepted February 2021 \\
Available online April 2021 \\
\hline JEL Classification \\
C99, I29, J15, L63, L86 \\
Keywords: \\
Computer-assisted teaching, \\
Educational tool, Arrays, Graphical \\
User Interface
\end{tabular}

\begin{abstract}
A B S T R A C T
Today's technological education has acquired new valences and has become a fundamental component of a modern educational process, whose purpose is to contribute to supporting the educational act itself. The use of computers and specific information applications in the educational process contributes substantially to increasing the interactivity of the educational processes to which all the actors involved are connected. Learning based on adequate computer tools integrates into the context of other means used in the educational process, amplifying their instructive-formative valences and contributes to the qualitative and quantitative improvement of the existing methods and techniques in education, at any level. Thus, this paper presents a component of an information system, developed to provide interactive support in understanding how to remove a given item from a onedimensional array.
\end{abstract}

\section{Introduction}

Although several decades have passed since computers have become an integral part of the educational process, information technologies continue to be perceived only as an alternative to a dynamic, modern education that provides the actors involved (i.e. students and teachers) with the specific tools which indisputably improve the teaching/learning process.

Today, one of the major consequences of COVID 19 was the rapid shift towards online education, which demonstrated that computer-assisted teaching activities represent an opportunity of the current educational society, whose total integration becomes a necessity.

However, distance learning (via online platforms) is not always a guarantee of a qualitative educational process. In this respect, it is important for teachers to select/choose modern and interactive solutions through which to achieve a diversification of the ways of transmitting the teaching content. In this context, information technologies represent an important support, able to contribute to the foundation of understanding the specific teaching content, which can sometimes prove to be abstract and challenging, even under normal circumstances of traditional educational systems.

\section{A brief description of C\# and WFA}

Computer applications for the educational process are computer programs written in a programming language, developed for use in the foundation of learning scenarios. Thus, this paper presents a component of such a computer application, developed in the Visual Studio C\# programming language (via Windows Forms Application - WFA) and whose purpose is to provide interactive (visual) support in understanding how to remove an item from a one-dimensional array. The main purpose is to present step by step, in the form of a graphic animation, the way in which the projected algorithm leads to the deletion of an element from a onedimensional array, describing the source code sequences through which this processing is carried out.

As described in [1], C\# is a high-level object-oriented and general-purpose programming language, created and developed by Microsoft, which consists of a set of definitions in classes that contain methods and the methods contain the program logic, i.e. the instructions which the computer executes [4]. C\# contains many innovative features and has been characterized as being a component-oriented language because it contains integral support for the writing of software components (features that directly support constituents of components, such as properties, methods and events) [6]. All components of the C \# language are associated with the notion of class, but also offer special types such as: interfaces, delegations or generic classes [2]. 
Windows Forms Application is a .NET framework and a graphical user interface that allows the development of form-based applications using a graphic designer. It includes a complete set of controls useful in designing any type of processing desired. Also, WFA allows to create graphic design applications that are very easy to deploy or update when necessary. Due to these features, the application that simulates the deletion of an item from a one-dimensional array was developed through this software tool.

\section{Some theoretical aspects related to removing an item from a one-dimensional array}

Removing an element from a vector can be quite common in practice: we have a list of students in a group of students and a student is expelled. How do we update the list of students? Thus, the problem of removing an item from a vector can be described as follows: give a string $\mathrm{S}$ (group of students) with $\mathrm{N}$ items (the number of students in that list) and an item $\mathrm{X}$. Thus, the problem involves removing from the string $\mathrm{S}$ the item with the value $\mathrm{X}$ (our expelled student, for example).

The method we can use in order to perform deletion, involves (initially) identifying the position in the vector where the element to be removed is located. Once its position is identified (we assume that it is in position $\mathrm{k}$ ), the deletion algorithm involves moving all items with a forward position (the first moved item is the one in position $\mathrm{k}+1$ which is moved to position $\mathrm{k}$ ) and then removing the last item of the vector. Practically, after moving an item, its value will be found in the vector on two positions: position i and position $\mathrm{i}+1$. Thus, removing the last item of the vector (performed after the last move of items) will remove one of the two duplicate values from the vector [3].

In this sense, the aim of the paper is to make an animated graphic representation of how an item is removed by moving it to its previous position. At each step, the moved element (by an animation) is marked with the Light Blue color; after moving it to the previous position, it is marked with the Lawn Green color, and at the end, the item from the last position which will be removed by resizing the vector, is marked with the Orange color.

\section{Animated software solution for understanding the removing of items from an array}

Figure 1 shows the graphical interface of the software application for removing an item from a vector. Initially, the vector contains ten elements (10 is the value of a local variable) that were randomly generated through the Random Class (the application also includes a component through which new elements can be inserted into the vector). Random generation of vector elements and their storage in the onedimensional array is done by calling the static constructor of the static class ArrayClass (figure 2).

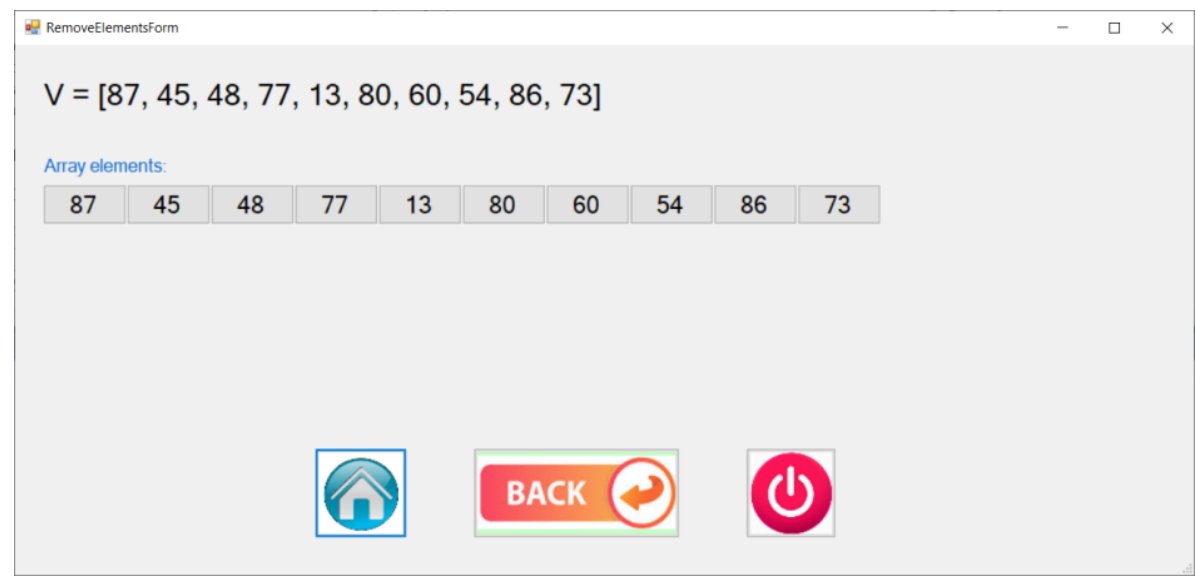

Figure 1. Graphical interface of the software application for removing an item from a vector

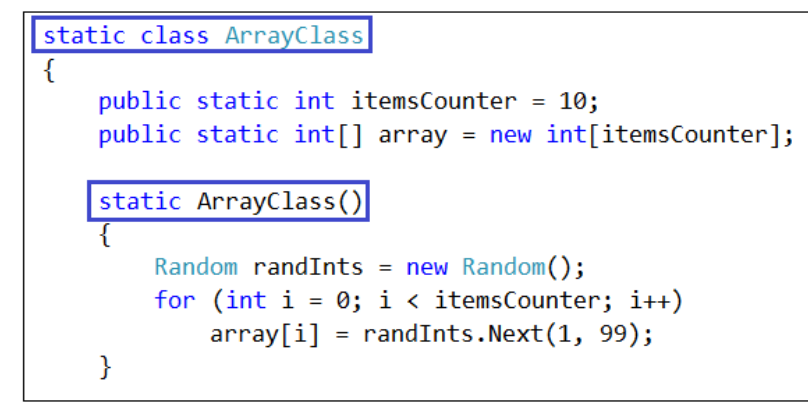

Figure 2. Source code for random generation of array elements

As shown in Figure 1, the items of the array are displayed on the graphical interface at the top of it. This is done by using another method of the ArrayClass class (figure 3), which has a formal Label formal 
parameter, because the items of the array are displayed in a Label control. In this way, at each method call, the items of the array are displayed in the Label control transmitted as a parameter. The local variable itemCounter permanently stores the current size of the array; if this size is 0 , it means that the array has no elements and the corresponding message will be displayed: "The array has no elements!".

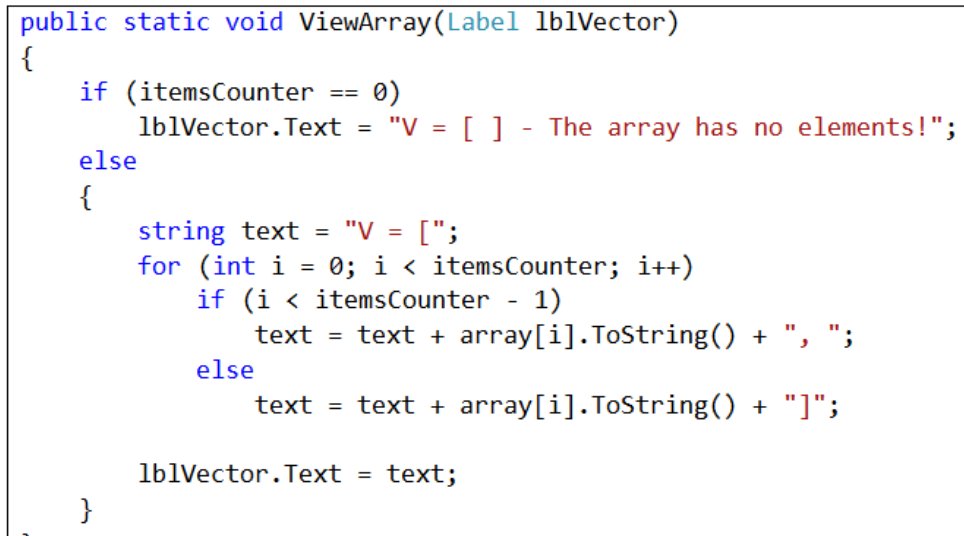

Figure 3. ViewArray Method

Moreover, in figure 1 it can be seen that the application generates buttons for each item of the array. When the user wants to delete an element, all they have to do is press (click) the button that corresponds to the item in the vector which will be deleted. Creating the buttons corresponding to the items in the array is done by calling the createButton method which is defined in the same ArrayClass class (figure 4). The method has three formal parameters: a string parameter that receives (when the method is called) the value of the item on the new button, and two other integer parameters, which have the role of specifying the position on the form where the button will be placed. Thus the buttons corresponding to the current items within the vector will be created (dynamically, during the execution of the application).

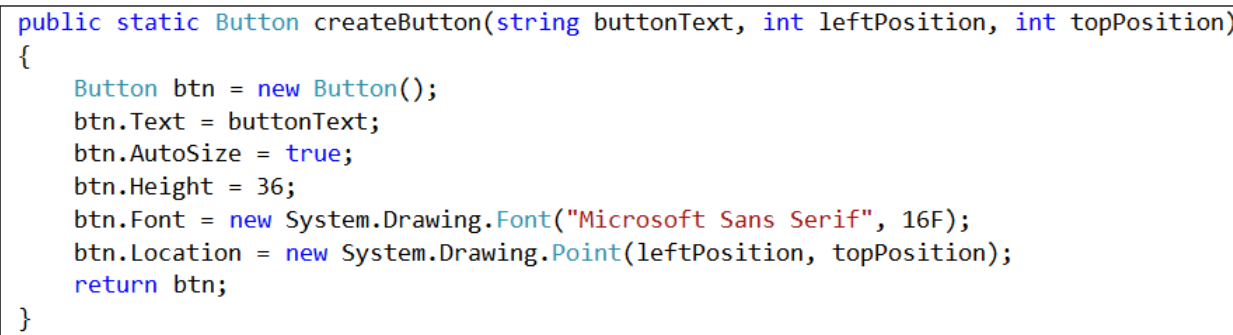

Figure 4 - createButton Method

As I mentioned before, removing an item will be done by pressing the corresponding button related to the item which will be deleted. Thus, in the example shown in Figure 5, we have/present the scenario in which the user wants to delete the item with the value 80 . To avoid accidentally deleting an item, the deletion is not done directly, but only after confirmation from the user. Thus, a message is displayed on the screen in which the user is notified that an item will be deleted and is asked to confirm this processing (pressing the OK button). If the Cancel button is pressed, then the deletion will no longer be performed. If the OK button is pressed, then the item deletion process begins (Figure 6).

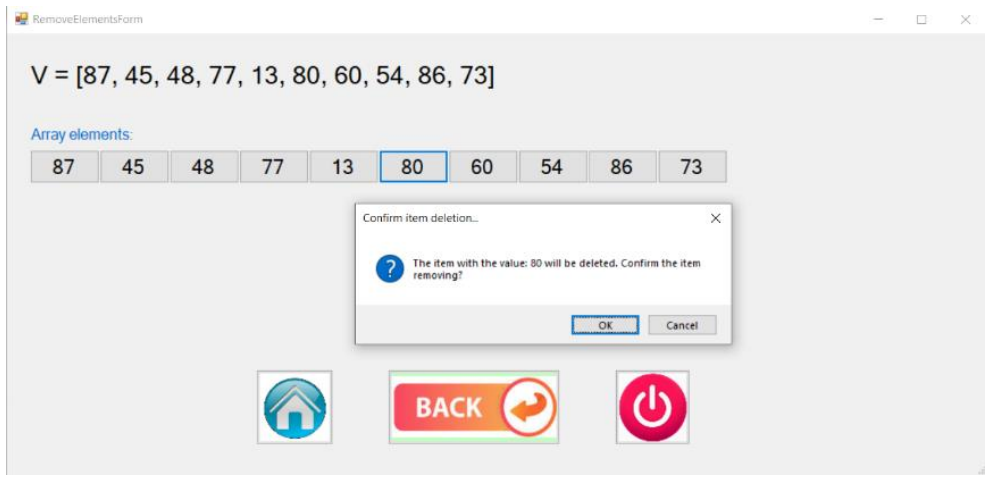

Figure 5. Confirmation request for deleting an item 


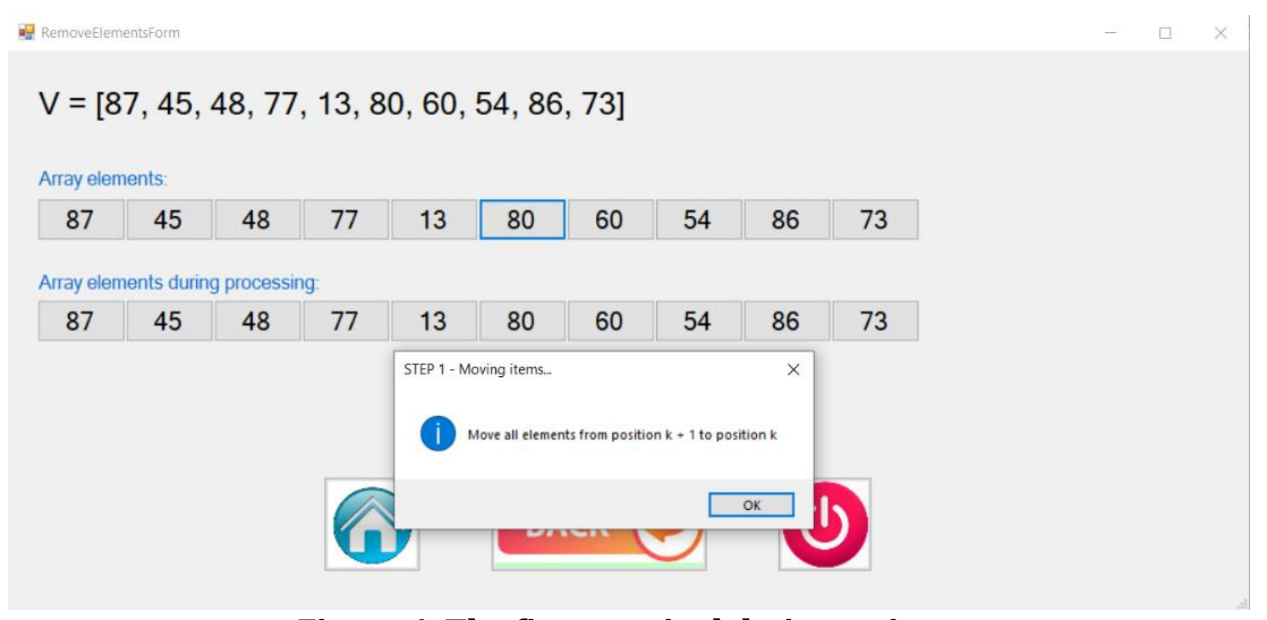

Figure 6. The first step in deleting an item

As shown in Figure 6, the simulation of the deletion process is triggered and a copy of the vector is displayed on the screen; the representation of the deletion of the item will be made in this second vector, in order to see in parallel, the initial content of the array and its modified content after each processing performed.

The deletion starts by pressing the OK button and involves moving all the items to the left with one position. For example, this process involves first moving the item which has the value 60 over the item with the value 80 , the one with the value 54 on the position of the item with the value 60 , and so on until moving the item from the last position (element 73) on the position of the item in front of it (on the position of the item with the value 86). By moving the item with the value 60 over the item with the value 80 , it basically means losing the contents of the item with the value 80 , which is equivalent to deleting it from the array.

Figure 7 Shows how to move the first item (item with value 60 instead of item with value 80 ).

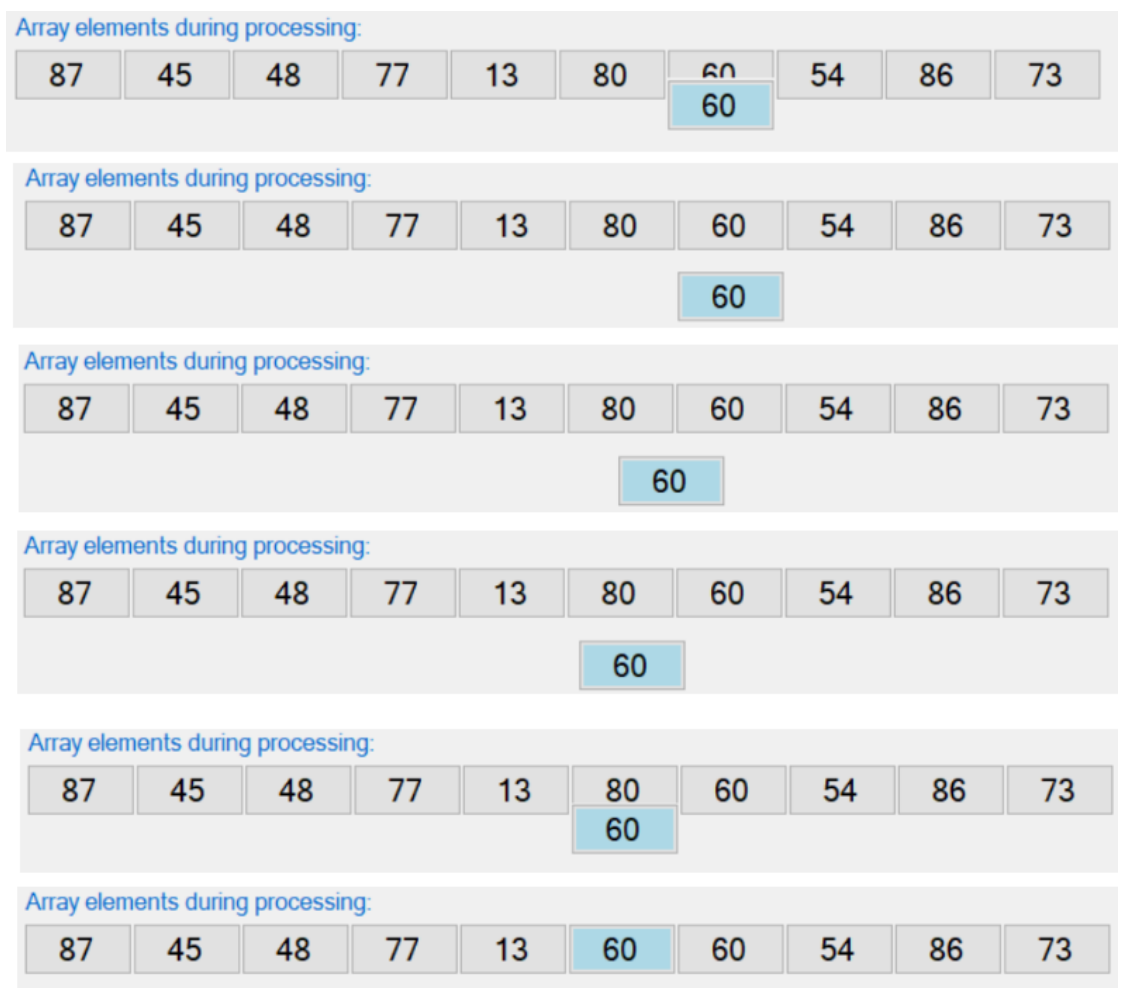

\section{Figure 7. Moving the item with value 60 instead of item with value 80 in the array}

The same procedure will be followed to move all items to the left with one position. After completing this process, all moved items will be represented in the second array with the Lawn Green color, and the last item is represented with the Orange color (it is the item that will be removed from the array) - Figure 8. A corresponding message will be displayed, informing the user about the position from which the items were moved (position 5 in our example). The user is also informed that the array will be resized. 


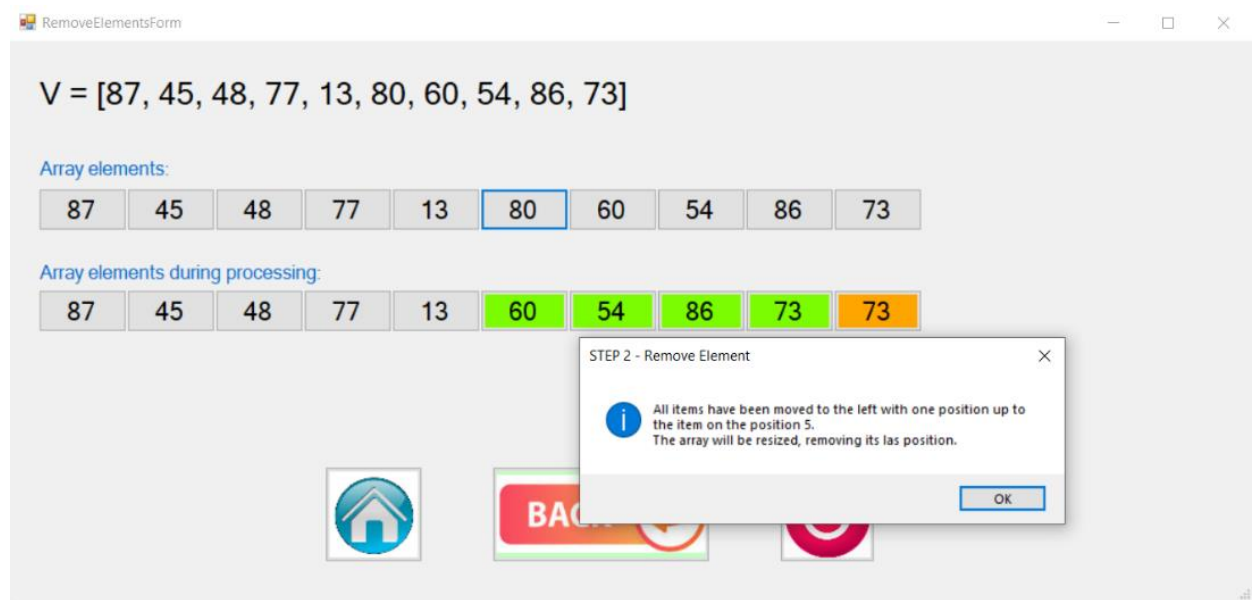

Figure 8. The second step in deleting an item - resizing array

In Figure 9 it can be seen that the item was removed from the second array by moving all the elements from after the item selected for deletion (the item with the value 80 ) to the left by one position. Then, the array is updated and displayed with the new content (Figure 10) and a new processing can be initiated (we can start deleting another element).

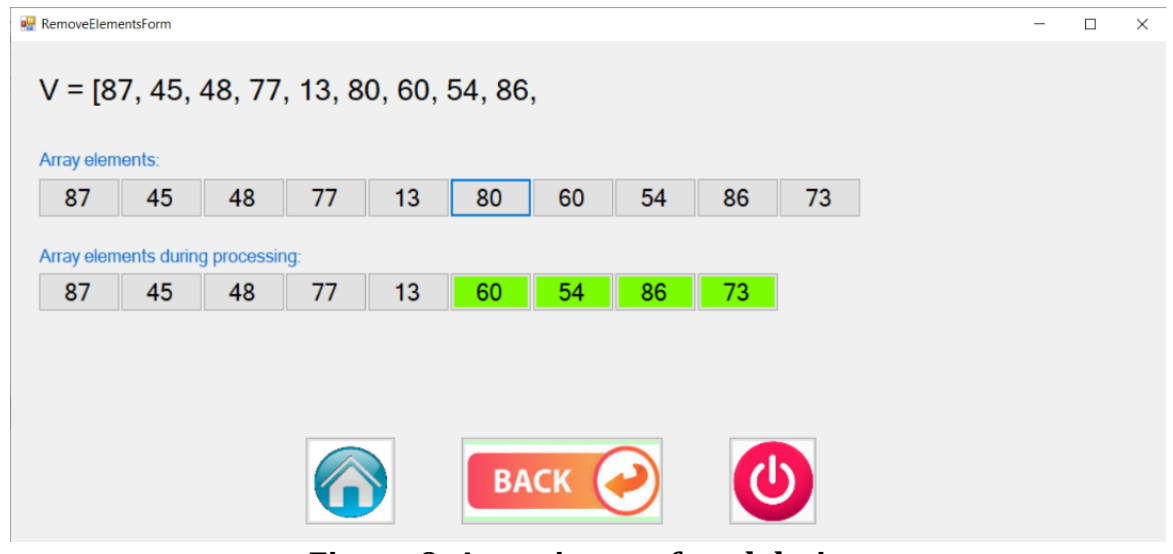

Figure 9. Array items after deletion

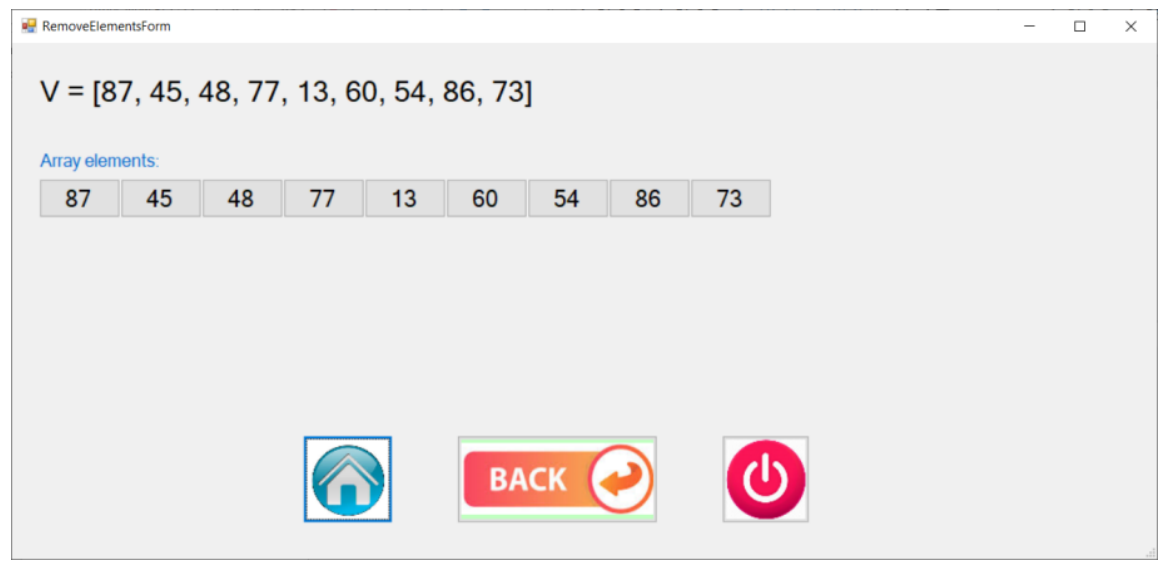

Figure 10. Graphical interface with the updated array for a new processing

\section{Conclusions and final remarks}

This paper presents a component of an information system, which was developed to provide interactive support in understanding how to remove an element from a one-dimensional array. This solution could be used as an educational tool in the didactic activity, being able to contribute to the proper understanding of how to process an array (from the perspective of deleting an item from an array). The purpose of developing such an information system was related to trying to provide interactive support in understanding how to remove items from a one-dimensional array. Thus, the paper presents in the form of a graphic animation the way in which an element is removed from a one-dimensional array, being described including source code sequences through which this processing is performed. 


\section{References}

1. Aho, A.V., Hopcroft, J.H., Ullman, J.D. (1988). Data Structures and Algorithms, Addison Wesley Publishing Company Kendal, S. (2011). Object Oriented Programming using C\#, eBooks and textbooks from bookboon.com.

Knuth, D.E. (1976). Tratat de programarea calculatoarelor, Sortare și căutare, vol. III, Editura Tehnică, București.

4. Nakov, S., Kolev, V. \& Co. (2013). Fundamentals of computer programming with Csharp, Telerik Software Academy.

5. Purdum, J. (2012). Beginning Object Oriented Programming with C\#, Wrox, John Wiley \& Sons

6. Schildt, H. (2009). C\# 3.0: A Beginner's Guide, Mc Graw Hill. 